\title{
An adaptation-level theory account of a relative-size illusion'
}

\author{
FRANK RESTLE and COLEMAN T. MERRYMAN, Indiana \\ University, Bloomington, Ind. 47401
}

A horizontal line (L) looks shorter with large boxes $(B)$ at the ends than with small Bs. This illusion was measured by having Ss judge the length of $L$ on a 6-point scale. Six different lengths of $L$ were combined factorially with six sizes of $B$ to form 36 stimuli, and $51 \mathrm{Ss}$ made four judgments each. Mean judgment increased smoothly with $L$ and decreased with $B$. Data were fitted using Adaptation-Level formulas, and the weight of $B$ was found to be approximately $14 \%$. However, $B$ had almost no effect on the judgment of very short Ls.

The full theoretical significance of geometrical illusions is obscured by the lack of accurate measurements of their magnitudes. The main methods of measurement call upon $S$ to compare two displays, each of which may be relatively complex, and which may interact. The point of subjective equality is usually well defined experimentally, but it is the product of many complex factors.

This paper reports measurements of a familiar relative-size illusion by the method of single stimuli. On each trial, a display like that shown in Fig. 1 is presented, and $S$ judges the length of the line (L) between boxes (B). The effect of B can therefore be measured relative to the effect of $L$, and one can estimate the change in $L$ required to compensate for the effects of a given change in B. Thus, a measurement of the illusion is obtained in physical amounts.

Adaptation-Level (AL) theory (Helson, 1964) states that the size of an object is judged relative to the sizes of other objects in the immediate surround. The fundamental equation is $J_{A}(X)=X / A$, where $A$ is the adaptation level, $X$ is the physical size of the object to be judged, and $J_{A}(X)$ is the judged size of $X$, given adaptation level $\mathrm{A}$. $\mathrm{A}$ is defined as the weighted geometric mean of all stimulus objects in the field.

When applied to the display in Fig. 1, the equation becomes $J_{A}(L)=L / A$, where $A$ is the weighted geometric mean of $L, B$, and other factors, $K$, which are either constant or vary randomly from trial to trial. Let the weights of $L, B$, and $K$, be, respectively, $w_{L}, w_{B}$, and $w_{K}$. Then

$$
A=\left(L^{w_{L}} B^{w_{B}} K^{w_{K}}\right) \overline{w_{L}+w_{B}+w_{K}} .
$$

Since the weights are in arbitrary units, they can be normalized so that their sum is unity. Then,

$$
\text { and } \begin{aligned}
& A=\left(L^{w_{L}} B^{w_{B}} K^{w_{K}}\right), \\
& J_{A}(L)=\frac{L}{A}=L^{1-w_{L}} B^{-w_{B}} K^{-w_{K}} .
\end{aligned}
$$

If the assumption is made that $S$ 's response is a linear function of $\mathrm{J}_{\mathrm{A}}$ (L) (a linear response rule), then $\mathrm{w}_{\mathrm{L}}$ and $\mathrm{w}_{\mathrm{B}}$ may be estimated and quantitative predictions made. The present study was performed to assess the ability of the $\mathrm{AL}$ theory with the linear response rule to handle the results of the simple relative-size illusion experiment.

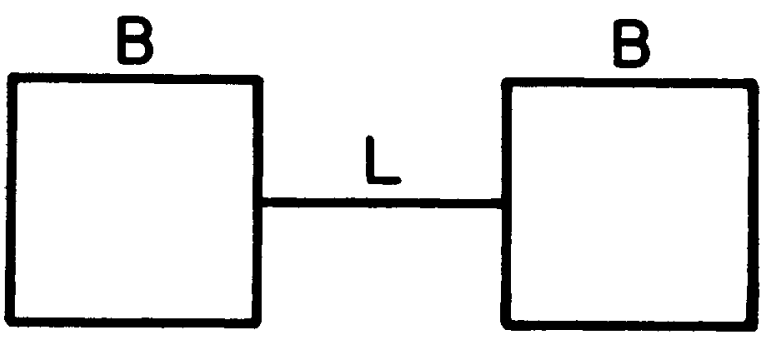

Fig. 1. An example of the stimuli used in the experiment.

\section{SUBJECTS}

Fifty-one students from introductory psychology courses at Indiana University served as part of course requirements.

$$
\text { APPARATUS }
$$

The Ss were run in groups of up to four at a time, seated side by side in booths separated by plywood dividers. Before each $\mathrm{S}$ was a grey metal box with six red response buttons. Approximately $8 \mathrm{ft}$ in front of the Ss was a white screen.

Stimuli consisted of 36 slides, each showing a white horizontal line with a white outline of a square on either end. The background of the slide was black. Six line lengths $(4,6,8,10,12$, and 14 units) and six box sizes $(4,6,8,10,12$, and 14 units on each side) were combined factorially to form the 36 slides. Each unit corresponded to approximately $1 \mathrm{~cm}$.

PROCEDURE

After the Ss had been seated, the following instructions were read: "We are interested in how appearances of lengths of lines vary with changes in background. I am going to show you some slides of horizontal lines with squares on either end. I want you to tell me how long the lines seem to you. You will tell me by pressing one of the six red buttons on the box in front of you. If the line seems quite short, you are to press the left button; if it seems quite long, you are to press the right button. The other four buttons correspond to intermediate lengths."

The 36 slides were then presented one at a time in random order, a different random order for each group of Ss. After all Ss had responded to a slide, there was a 5 -sec intertrial interval, after which a new slide was presented. This procedure continued until all Ss had seen and responded to each slide five times. The first time through the 36 slides was treated as practice, and only the last four responses to each slide were used in the analysis.

RESULTS

The six buttons from left to right were assigned the numbers one through six, respectively.

The mean response to each slide was calculated over Ss. Figure 2 shows the obtained and predicted mean responses. The theoretical curves were based on least squares estimates of three

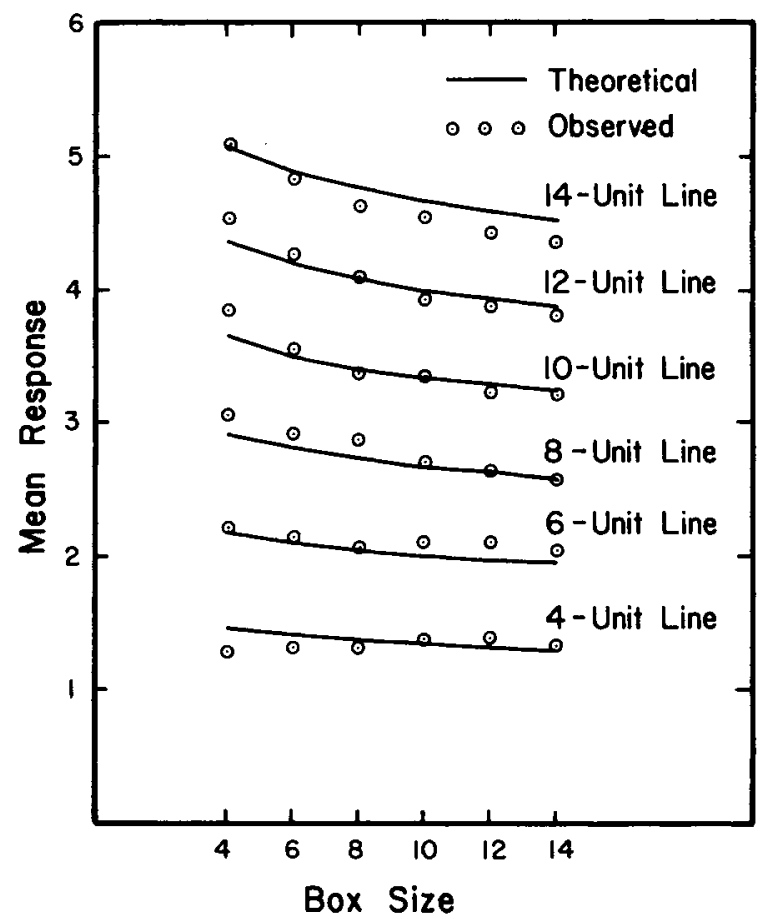

Fig. 2. Observed and predicted responses for each stimulus. 


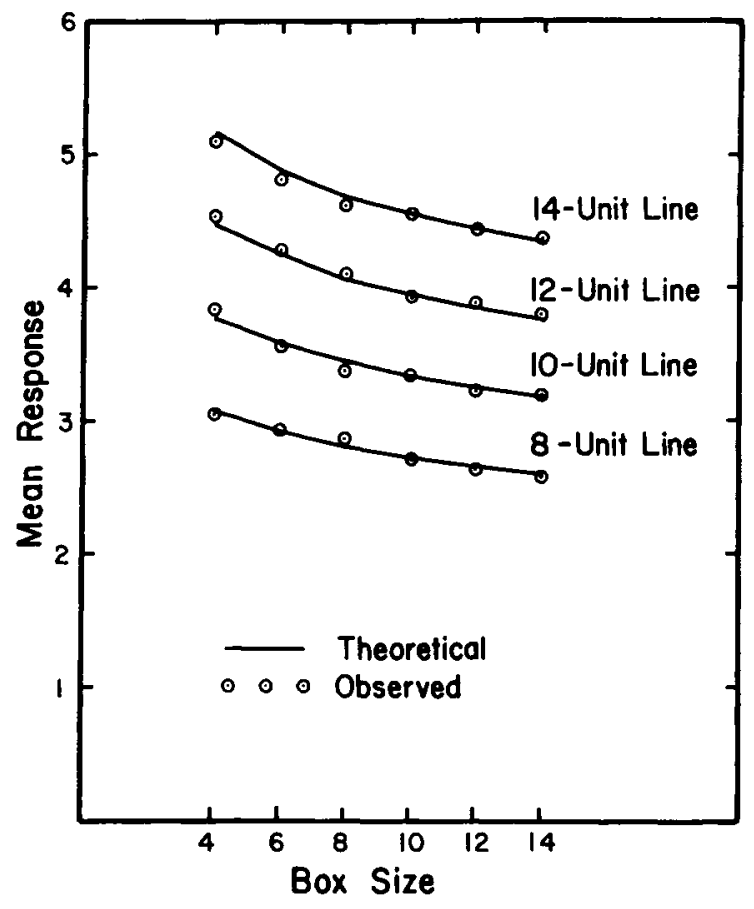

Fig. 3. Observed and predicted responses for the longest four lines.

parameters, ${ }^{2} w_{L}, w_{B}$, and $C$, where $C$ is a function of $K$. The values of $w_{L}, w_{B}$, and $C$ were $.007, .093$, and .867 , respectively.

The judgments of Ls of length $8,10,12$, and 14 decrease systematically as $B$ increases. Ls of length 4 and 6 do not change in their judged length as B varies. The result, shown in Fig. 2, is that the predicted illusion curves are too flat for the longer Ls and are too steep for the very short Ls.

An analysis was performed using only the Ls of length 8,10 , 12 , and 14 . The new parameter estimates were $.082, .138$, and
.593 for $w_{L}, w_{B}$, and $C$, respectively. The fit of the theory to this part of the data is shown in Fig. 3.

In summary, the data agree closely with the AL model in showing a relative-size illusion, provided that $L$ is 8 to 14 units long, as B varies from 4 to 14 units in height. No previous studies known to the authors have shown that this illusion disappears when $L$ is short. Supplementary studies suggest that this is not a response "floor" effect, but we have not been able to determine why short Ls are not affected in apparent size by the size of B. DISCUSSION

The results of this experiment show that the relative-size illusion can be demonstrated, with no comparative judgments, by the method of single stimuli. The measurements reveal that for Ls of length $8,10,12$, and 14 units, the judgment of a given $L$ decreases systematically as $B$ increases, roughly as a power function, $J(L) \propto B^{-W_{B}}$. This finding agrees with the $A L$ theory that $\mathrm{L}$ is being judged relative to its $\mathrm{AL}$ and that $\mathrm{AL}$ is a weighted geometric mean of all factors.

In this case, as in many similar illusions, it is not strictly accurate to measure the illusion in percentages, for the percentage change in $J_{A}(L)$ depends on the variation in $B$ that is used. However, given the $\mathrm{AL}$ equation, and knowing the sizes of $\mathrm{B}$ employed, it is possible to calculate a percentage illusion (I) by the equation $I=100\left(J_{2}-J_{1}\right) / J_{1}$, where $J_{1}$ and $J_{2}$ are two judgments of the same $L$ with different box sizes, $B_{1}$ and $B_{2}$. Then,

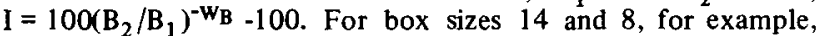
$\mathrm{B}_{2} / \mathrm{B}_{1}=.571$, and the calculated illusion, based on .138 as the estimate of $\mathrm{w}_{B}$, is $8 \%$.

The AL formulation permits reasonable extrapolation to other sizes of Bs, and in effect reduces the whole mass of observations to the self-adaptation parameter $\left(w_{L}\right)$, the box-influence parameter $\left(w_{B}\right)$, and one other fitted constant $(C)$. The magnitude of the illusion is represented in $w_{B}$.

\section{REFERENCES}

HELSON, H. Adaptation-level theory. New York: Harper \& Row, 1964. NOTE

1. This research was supported by National Science Foundation Grant GB 2848 and Public Health Service Grant MH-12541 to the senior author. The authors wish to thank David Lloyd for preparing the stimuli.

2. These estimates were based on the assumption that the response is proportional to the judgment (special case of the linear response rule). 